\title{
Patient and Physician Preferences for Therapy Characteristics for Psoriasis: A Discrete Choice Experiment in Japan
}

\author{
Timothy Bolt ${ }^{1} \cdot$ Hisanori Kobayashi ${ }^{2} \cdot$ Jörg Mahlich ${ }^{3,4}(0)$
}

Published online: 30 October 2018

(c) The Author(s) 2018

\begin{abstract}
Background With progress being made in the treatment of psoriasis, a variety of clinical research and treatment options are being pursued. This study used a discrete choice experiment (DCE) to estimate treatment characteristic preferences for both patients and physicians in Japan. Subgroup analysis was also applied in order to examine differences within the range of patients and within the range of physicians.

Methods The DCE was developed with the input of clinical experts in the treatment of psoriasis to ensure inclusion of the most relevant attributes at appropriate levels in a way that is understandable to both physicians and patients. The study was conducted on parallel samples of Japanese physicians $(n=161)$ and Japanese psoriasis patients $(n=306)$ through an online panel. For each sample, a conditional logit statistical model and subgroup analysis were then performed to estimate respondent preferences for treatment attributes.

Results The overall findings are that better treatment efficacy as measured by proportion of patients achieving $90 \%$ reduction in the Psoriasis Area and Severity Index score (PASI 90), lower risk of adverse events and the availability of a bio-holiday are important decision factors for both patients and physicians. Low injection frequency is less of a priority for both samples. Also, while both groups demonstrate a preference to receive the treatment injections at a clinic by a healthcare professional rather than self-injection at home, this is more pronounced for the patient sample. The physician sample shows considerably more emphasis on the type of injection, though both samples prefer subcutaneous injections to intravenous injections. Implications This study reveals the importance of addressing both clinical effectiveness and process factors in systemic, non-topical psoriasis treatments to gain acceptance by both physicians and patients. As well as efficacy (as measured by PASI 90), which remains a consistent priority in treatment, administration and development of new treatments should also consider process factors such as the mode of administration and possibility for a bio-holiday.
\end{abstract}

Electronic supplementary material The online version of this article (https://doi.org/10.1007/s41669-018-0104-1) contains supplementary material, which is available to authorized users.

Timothy Bolt

tbolt@mail.saitama-u.ac.jp

1 Faculty of Economics, Saitama University, Saitama, Japan

2 Medical Affairs, Janssen Pharmaceutical K.K., Tokyo, Japan

3 Health Economics and Outcomes Research, Janssen, Neuss, Germany

4 Düsseldorf Institute for Competition Economics, University of Düsseldorf, Düsseldorf, Germany

\section{Key Points for Decision Makers}

Patients and physicians are willing to trade-off treatment efficacy for preferred process factors (injection frequency, injection mode, availability of bio-holiday breaks in treatment).

Efficacy (as measured by PASI 90 and reduction of adverse events) remains a consistent priority in treatment among both physicians and patients and is stronger for patients.

Patients are more focused on a few key treatment attributes (effectiveness, treatment setting, availability of a bio-holiday) while physicians' responses are more balanced across attributes. 


\section{Introduction}

Psoriasis is a chronic, systemic autoimmune disease that affects the skin and may be associated with other inflammatory conditions and comorbidities. The prevalence of diagnosed psoriasis has previously been estimated to be $0.34 \%$ in Japan [1], which is much lower than in either the US (3.4\%) [2] or Europe (from $0.7 \%$ in Scotland to $8 \%$ in Norway) [3]. The differences can largely be attributed to the higher prevalence of obesity in the West, since this has been determined to be a significant risk factor [4]. Lack of vitamin D can also be partially responsible for high prevalence rates in northern countries [5].

The burden of psoriasis is substantial and the condition has a significant negative impact on patients' quality of life. Psoriasis has been linked to depression and suicidal tendencies among patients. The costs associated with the decrease in quality of life, lost productivity and work absenteeism may be enormous [6].

Treatment options depend on disease severity and include topical therapies such as creams, phototherapies for mild forms and systemic agents such as methotrexate for more severe forms of psoriasis. The development of biological agents targeting tumour necrosis factor alpha (TNF $\alpha$ ), interleukin (IL)-17, IL-12 and IL-23 has changed the treatment paradigm for severe psoriasis. Biologic agents can be administered through subcutaneous injections or intravenous infusions and have demonstrated impressive efficacy [7]. A recent survey of psoriasis patients reported that overall patient satisfaction with existing psoriasis therapies was only modest; however, those treated with biologic agents exhibited highest treatment satisfaction over oral therapy, phototherapy and topical therapy [8]. In Japan, a recent analysis of claims data reported that out of 28,006 patients with a diagnosis of psoriasis, 3093 (11\%) received this kind of treatment [9], which is similar to the proportion found for patients in the US [10]. A possible reason why biologics are not prescribed more widely might be their high cost. In Japan, yearly cost per PASI 75 responder (those with a $75 \%$ reduction in their Psoriasis Area and Severity Index score) was estimated to be between JPY 1.5 million and JPY 7.4 million ( US \$15,000-US \$74,000) [11]. A study from the US found that treatment dissatisfaction was related to high cost [12].

In Japan, seven biologics for treating psoriasis are available: infliximab and adalimumab were approved in 2010, ustekinumab was approved in 2011, secukinumab was approved in 2014, brodalumab and ixekizumab were approved in 2016 and guselkumab was approved in 2018. Although the development of these treatment options raises the expectation of treatment success from the previous levels (i.e. PASI 75) to higher levels (i.e. PASI 90), they all have different profiles regarding efficacy, safety, frequency and mode of administration.

Discrete choice experiments (DCEs) are a set of stated preference, questionnaire-based methods that enable researchers to statistically estimate how respondents' value different aspects of services. This is done through asking study subjects to choose between sets of hypothetical options with different attributes (characteristics) at specified levels in a series of presented choices. DCEs are characterized by statistically modelling the respondents' utility maximizing trade-offs based on the levels of the attributes of the options presented. To date, patient and physician treatment preferences have been studied in a number of indications and are found to be particularly useful for considering both health outcome and process factors [13-18].

This study involves eliciting both patient and physician preferences for such treatment attributes by means of a DCE. Previous DCEs in psoriasis have evaluated either physician [19] or patient [20] preferences for treatment attributes, with few studies comparing the concordance of the preferences between stakeholders [21, 22]. For Japan, to the best of our knowledge, this would be the first attempt to compare physician and patient preferences in psoriasis. Furthermore, subgroups within each of these samples were then tested for any statistically significant differences in treatment priorities and preferences.

\section{Methods}

\subsection{Discrete Choice Experiment Development}

This study opted to use a DCE to identify the patient and physician priorities from among possible health outcomes and experience factors in the treatment of psoriasis. The DCE method enables respondents to consider and express preferences for both real and hypothetical options based on the characteristics of the choices. From statistically modelling which choices are made by the respondents, the relative value of the characteristics can be estimated. As is recognized in the DCE literature and across good practice guidelines, attribute and level selection within a DCE study is a critical step, not just for ensuring inclusion of relevant attributes but also to ensure the levels that will reflect the decision of interest and induce trade-offs among the choice sets for the respondents [23, 24]. This study's DCE included attributes and levels that were selected through a workshop with eight specialist clinicians. The process was facilitated by a study member who had also undertaken a literature review to identify a full list of potential attributes. While health states were considered as an outcome measure, ultimately the focus was on standard clinical outcomes for psoriasis 
treatment and aspects of the experience of receiving treatment. For the appropriate levels, the panel was instructed to anchor this in current treatment options and near future possible improvements. The finally selected seven attributes and corresponding levels for these are listed in Table 1.

The seven attributes and corresponding levels $\left(4^{\wedge} 4 * 2^{\wedge} 3\right)$ were reduced to 16 choice sets in SAS software, version 9.4 (SAS Institute, Cary, NC, USA) using the MktEx macro [25]. The resulting orthogonal partial factorial main effects design had a reported relative D-efficiency of $100 \%$. A mod shift approach, in which each attribute had its level varied by a set value, was used to generate the alternative option for each of the 16 choice sets from the original experimental design.

Visual representations of the attribute levels were presented along with the quantitative or textual description of the choice option to reduce the cognitive burden of respondents and increase engagement. These were designed such that each attribute was of roughly equal visual impact, as shown in Fig. 1. As this was an online-administered questionnaire, the sequence in which the 16 choice pairs were presented to each respondent was random to avoid any systematic biases from the question sequence.

\subsection{DCE Administration and Analysis}

The questionnaire was piloted with a patient sample $(n=31)$ and physician sample $(n=33)$ to check that trade-offs were being made in the treatment choice selection and also to check that the respondent populations could understand presented treatment options (responding positively to a follow-up question on their self-reported understanding of the presented choice options in the DCE: patients $87 \%$; physicians $94 \%$ ).

The final questionnaire with the 16 choice sets was administered to screened samples from a physician panel $(n=161)$ and to a patient panel $(n=306)$ during November to December 2017. Among the inclusion criteria for the physicians was having to treat moderate or severe psoriasis patients each month. Patients had to be adults (18 years or older) who have had a diagnosis for at least a year and are actively receiving treatment. To exclude the mild cases for which the non-topical treatments are not typically prescribed, psoriasis severity screening questions were applied to the patient panel. To be included, patients had to meet at least one of the following criteria: current treatment is 'Oral Drug' or 'Biologic Agent' (non-topical, systemic treatments) or extent of body surface area (BSA) coverage of at least three 'hands' or self-reported severity of 'moderate' or 'severe'. In setting sample size requirements, the team followed the accepted guidelines from the literature considering the number of attributes, choices and planned sub-group comparisons for a main-effects study without attribute interactions [24, 26, 27]. Along with the guidelines, consideration was made of the expected variation in preferences across the patient sample as well as

Table 1 Attributes and levels

\begin{tabular}{ll}
\hline Attribute & Levels \\
\hline PASI 90 (percent of patients who achieve 90\% skin clearance) & $90 \%$ \\
& $80 \%$ \\
Stop rate for treatment (percentage of patients not completing treatment) & $60 \%$ \\
& $40 \%$ \\
Bio-holiday availability (6-month break in treatment without worsening of symptoms) & $5 \%$ \\
& $10 \%$ \\
Risk of severe adverse events (including serious infections) & No \\
& $0.2 \%$ \\
Injection frequency (number of injections in the first year of treatment) & $0.5 \%$ \\
& $2.0 \%$ \\
Injection type & $5.0 \%$ \\
Who provides injection? & 6 injections \\
& 8 injections \\
& 16 injections \\
& 26 injections \\
& IV (intravenous) \\
& SC (subcutaneous)
\end{tabular}

PASI 90 90\% reduction in the Psoriasis Area and Severity Index score 


\begin{tabular}{|c|c|c|}
\hline & Treatment A & Treatment B \\
\hline $\begin{array}{l}\text { Proportion of patients } \\
\text { who have at least } 90 \% \\
\text { skin clearance } \\
\text { compared to the start } \\
\text { of treatment }\end{array}$ & $\begin{array}{c}\text { Goal: } 90 \% \text { clearance } \\
40 \%:\end{array}$ & $\begin{array}{c}\text { Goal: } 90 \% \text { clearance } \\
\mathbf{8 0 \%} \text { out of } 10 \text { patients meet the goal. }\end{array}$ \\
\hline $\begin{array}{l}\text { Percentage of patients } \\
\text { instructed to stop } \\
\text { treatment due to loss } \\
\text { of efficacy or side- } \\
\text { effects }\end{array}$ & $\begin{array}{c}\text { 5\%: } \\
\text { 1 out of every } 20 \text { patients stops }\end{array}$ & $\begin{array}{c}\text { 80\%: } \\
8 \text { out of every } 20 \text { patients stops }\end{array}$ \\
\hline $\begin{array}{l}\text { Possibility of taking a } \\
\text { break at least } 6 \\
\text { months in treatment } \\
\text { without worsening of } \\
\text { symptoms }\end{array}$ & $\begin{array}{l}\text { It is not possible to take a break in } \\
\text { treatment without worsening of } \\
\text { symptoms. }\end{array}$ & $\begin{array}{l}\text { It is possible to take a break at least } 6 \\
\text { months in treatment without } \\
\text { worsening of symptoms. }\end{array}$ \\
\hline $\begin{array}{l}\text { Risk of severe side- } \\
\text { effects, including } \\
\text { serious infectious }\end{array}$ & $\begin{array}{c}c+\ldots=1 \\
2 \% \text { : } \\
\text { for every } 1000 \text { patients, } 20 \text { will have a } \\
\text { severe adverse event }\end{array}$ & $\begin{array}{c}\text { 5\%: } \\
\text { por every } 1000 \text { patients, } 50 \text { will have a } \\
\text { severe adverse event }\end{array}$ \\
\hline $\begin{array}{l}\text { Number of times of } \\
\text { injections in the } 1^{\text {st }} \\
\text { year }\end{array}$ & 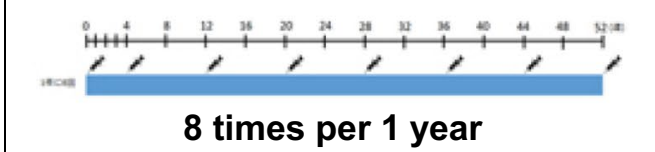 & 26 times per 1 year \\
\hline Injection type & Subcutaneous injection & Intravenous injection (IV) \\
\hline $\begin{array}{l}\text { Where \& by whom } \\
\text { injection is } \\
\text { administered }\end{array}$ & Health Care Professional at a clinic & By oneself at home \\
\hline
\end{tabular}

O Treatment A

O Treatment B

Fig. 1 Example of presented discrete choice experiment choice (simplified translation) 
Table 2 Sample characteristics

Physician sample $n=161$ Patient sample $n=306$

Gender

Male

$140(87.0 \%)$

$252(82.4 \%)$

Female

$21(13.0 \%)$

$54(17.6 \%)$

Age

49.4 years $(9.2)$

$53.4(10.7)$

Physicians

How many psoriasis patients do you treat per month?

Psoriasis vulgaris patients

Arthropathic vulgaris patients

$6.14(10.82)$

Other vulgaris patients

$2.68(5.6)$

Physician's speciality

General practitioner

$36(22.4 \%)$

Dermatologist

$93(57.8 \%)$

Rheumatologist

$12(7.5 \%)$

Orthopaedist

$18(11.2 \%)$

Other

$2(1.2 \%)$

How long have you practiced?

21.8 years $(8.1$ years $)$

Patients

What is the highest level of education you have completed?

High school

$91(29.7 \%)$

Technical/vocational college

$43(14.1 \%)$

University-Bachelor degree

$151(49.3 \%)$

University-Postgrad degree

$10(3.3 \%)$

Other

$11(3.6 \%)$

Type of psoriasis

Psoriasis

$279(91.2 \%)$

Psoriatic arthritis

$46(15.0 \%)$

Generalized pustular psoriasis

$6(2.0 \%)$

Other psoriasis

$6(2.0 \%)$

Self-rated severity of psoriasis (considering the symptom intensity, body surface area coverage and effect on daily activities)

Mild

$153(50.0 \%)$

Moderate

$114(37.3 \%)$

Severe

$31(10.1 \%)$

I don't know

$8(2.6 \%)$

Currently monthly spend on psoriasis treatment

8520 JPY $(17,600$ JPY $)$

How many hand-sized areas of psoriasis symptoms on body

$10.7(17.2)$

Importance of being involved (express opinion, make recommendation, etc.) when deciding treatment options

Very important

$197(64.4 \%)$

Somewhat important

$93(30.4 \%)$

Not that important

$15(4.9 \%)$

Not at all important

$1(0.3 \%)$

Degree to which doctor is perceived to take patient opinion/recommendations into consideration in treatment decisions

Highly into consideration

Somewhat into consideration

Not much into consideration

Not at all into consideration

$3(1.0 \%)$

Categorical variables presented as $n(\%)$

Continuous variables presented as: mean (SD) 
practical constraints in the number of physicians meeting inclusion criteria (Table 2).

After data cleaning, the choice data and respondent socioeconomic variables were imported into STATA v.14 for analysis. The DCE analysis was undertaken with a conditional logit model in which each of the three 4-level attributes (PASI90, StopRate, RiskAE) were continuous variables. The following utility specification, in which the $\beta$ coefficients reflected the impact of each attribute on the likelihood of choosing an offered treatment and $\varepsilon$ is the error term assumed to follow a Gumbel distribution, is estimated for this logit model:

$$
\begin{aligned}
U= & \alpha+\beta_{1} * \text { PASI90 }+\beta_{2} * \text { StopRate }+\beta_{3} * \text { BioHoliday } \\
& +\beta_{4} * \text { RiskAE }+\beta_{5} * \text { InjFrequency } \\
& +\beta_{6} * \text { InjType }+\beta_{7} * \text { InjWho }+\varepsilon .
\end{aligned}
$$

This study uses a conditional logit model to estimate the parameters of the DCE model for physicians (Table 3) and for patients (Table 4). (For ease of interpretation, these tables present the model results as both regression coefficients and as odds ratios for the attributes.) These tables also present each attribute as a 'Willingness to Accept Compensation' in terms of the key health outcome variables: PASI 90 and risk of adverse events (AEs).
The clinician workshop participants also identified key subgroups of interest within each sample to be analysed. Subgroup analysis within each of the two samples was conducted with Swait-Louviere Log-Likelihood Ratio tests [28]. For those sub-samples in which the S-L LL Ratio Test exceeds the $X^{2}$ test statistic, the utility models for the samples can be considered statistically different from one another.

\section{Results}

\subsection{Comparison of Physician and Patient Samples}

Descriptions of the respondents within the two samples are presented in Table 2.

The results for both the physician and patient samples demonstrate attribute preferences in the expected directions and are generally consistent with one another. The coefficient magnitudes suggest that better treatment efficacy (PASI 90), lower risk of AEs and also the availability of a bioholiday are important decision factors for both patients and physicians. Also, both groups prefer that the injections are administered at a clinic by a healthcare professional rather than being self-administered at home.
Table 3 Physician discrete choice experiment (DCE) results $(n=161)$
Table 4 Patient discrete choice experiment (DCE) results $(n=306)$

\begin{tabular}{lrllcc}
\hline & Coef. & Odds ratio & $P>z$ & WtAC PASI90 & WtAC RiskAE \\
\hline PASI 90 (per 10\% increase) & 0.1697 & 1.1849 & 0.0000 & -1.00 & 0.18 \\
Stop rate (per 5\% increase) & -0.0368 & 0.9639 & 0.0000 & 0.43 & -0.08 \\
Bio-holiday (Yes) & 0.1560 & 1.1689 & 0.0000 & -9.20 & 1.61 \\
Risk AE (per 1\% increase) & -0.0969 & 0.9077 & 0.0000 & 5.71 & -1.00 \\
Injection frequency (5 additional) & -0.0454 & 0.9556 & 0.0020 & 0.54 & -0.09 \\
Injection type (IV) & -0.4712 & 0.6243 & 0.0000 & 27.77 & -4.86 \\
Injection-who (HCP at clinic) & 0.0992 & 1.1043 & 0.0210 & -5.85 & 1.02 \\
\hline
\end{tabular}

$A E$ adverse events, $H C P$ healthcare provider, $I V$ intravenous, $P A S I 9090 \%$ reduction in the Psoriasis Area and Severity Index score, WtAC PASI90 the percentage changes in PASI 90 that would be needed to compensate for the change in the row attribute, WtAC RiskAE the percentage changes in RISK AE that would be needed to compensate for the change in the row attribute

\begin{tabular}{lrllcc}
\hline & \multicolumn{1}{c}{ Coef. } & Odds ratio & $P>z$ & WtAC PASI90 & WtAC RiskAE \\
\hline PASI 90 (per 10\% increase) & 0.1354 & 1.1450 & 0.0000 & -1.00 & 0.12 \\
Stop rate (per 5\% increase) & -0.0286 & 0.9718 & 0.0000 & 0.42 & -0.05 \\
Bio-holiday (Yes) & 0.1729 & 1.1888 & 0.0000 & -12.77 & 1.50 \\
Risk AE (per 1\% increase) & -0.1151 & 0.8913 & 0.0000 & 8.50 & -1.00 \\
Injection frequency (5 additional) & -0.1059 & 0.8995 & 0.0000 & 1.56 & -0.18 \\
Injection type (IV) & -0.0762 & 0.9266 & 0.0140 & 5.63 & -0.66 \\
Injection-who (HCP at clinic) & 0.3970 & 1.4874 & 0.0000 & -29.32 & 3.45 \\
\hline
\end{tabular}

$A E$ adverse events, $H C P$ healthcare provider, $I V$ intravenous, PASI $9090 \%$ reduction in the Psoriasis Area and Severity Index score, WtAC PASI90 the percentage changes in PASI90 which would be needed to compensate for the change in the row attribute, WtAC RiskAE the percentage changes in RISK AE would be needed to compensate for the change in the row attribute 
What is found to be much more important in the treatment choice decision among the physician sample than the patient sample is the 'injection type' attribute, in which physicians demonstrated preference for subcutaneous injections (SC) over intravenous injections (IV).

Patients tend to pay more attention to the "who administers the injection' attribute, preferring a healthcare professional at a clinic. Patients are also more likely to choose alternatives with a lower injection frequency.

The treatment choices made by the patient sample is more focused on certain key attributes, such as treatment effectiveness, the treatment setting ('InjWho') and availability of a 6-month bio-holiday, leading to strong results that are statistically significant. The full physician sample also has statistically significant results, but many of the actual size of impacts (coefficients values) are not that strong as a broader range of the attributes seem to be included in their trade-off considerations.

\subsection{Subgroup Analysis}

The biggest difference in priorities amongst the physicians is that general practitioners (GPs) put more weight on the treatment being delivered by a healthcare professional at a clinic and also more emphasis on the treatment effectiveness (PASI 90) than do the other physicians. Dermatologists indicate more focus on avoiding risk of adverse events, lower stop rates and the availability of a bio-holiday, with less relevance on the delivery setting.

Amongst the patients, those self-reporting as 'mild' psoriasis cases have similar preference for the effectiveness to those self-reporting as 'moderate' and 'severe' cases, but did have the injection type, risk attribute and injection setting attributes play a much larger role in their decisions (Table 5). (Alternative severity subgroup analysis was also undertaken using a body surface area measure criterion, the results for which are in Appendix A, see Electronic Supplementary Material.)

\section{Discussion}

Our results suggest that the treatment attributes regarding the possibility of a bio-holiday, efficacy in terms of PASI 90 and lower risk of AEs, are most important for both patients and physicians. Also, both groups prefer that medication is administered at a clinic instead of selfadministration at home. There is a strong aversion against

Table 5 Subgroup analysis

\begin{tabular}{|c|c|c|c|c|c|c|}
\hline & \multicolumn{4}{|l|}{ Physicians } & \multicolumn{2}{|c|}{ Patients: psoriasis severity } \\
\hline & $\begin{array}{l}\text { General practi- } \\
\text { tioners }\end{array}$ & Non-GPs & $\begin{array}{l}\text { Dermatologists } \\
\text { only }\end{array}$ & $\begin{array}{l}\text { Non-dermatolo- } \\
\text { gists }\end{array}$ & Mild patients only & $\begin{array}{l}\text { Moderate and severe } \\
\text { patients }\end{array}$ \\
\hline & $n=36$ & $n=125$ & $n=93$ & $n=68$ & $n=153$ & $n=145$ \\
\hline Attribute & Coefficient $(P>z)$ & Coefficient $(P>z)$ & Coefficient $(P>z)$ & Coefficient $(P>z)$ & Coefficient $(P>z)$ & Coefficient $(P>z)$ \\
\hline $\begin{array}{l}\text { PASI } 90 \text { (per } 10 \% \\
\text { increase) }\end{array}$ & $0.2197(0.000)$ & $0.1599(0.000)$ & $0.1571(0.000)$ & $0.1909(0.000)$ & $0.1358(0.000)$ & $0.1366(0.000)$ \\
\hline $\begin{array}{l}\text { Stop rate (per 5\% } \\
\text { increase) }\end{array}$ & $-0.0321(0.136)$ & - $0.0378(0.001)$ & $-0.0443(0.001)$ & $-0.0269(0.082)$ & $-0.0266(0.013)$ & $-0.0310(0.003)$ \\
\hline Bio-holiday (Yes) & $0.0087(0.927)$ & $0.1968(0.000)$ & $0.1719(0.002)$ & $0.1355(0.043)$ & $0.1630(0.000)$ & $0.1915(0.000)$ \\
\hline $\begin{array}{l}\text { Risk AE (per 1\% } \\
\text { increase) }\end{array}$ & $-0.0579(0.060)$ & $-0.1092(0.000)$ & $-0.1120(0.000)$ & $-0.0782(0.000)$ & $-0.1328(0.000)$ & $-0.0959(0.000)$ \\
\hline $\begin{array}{l}\text { Injection } \\
\text { frequency ( } 5 \\
\text { additional) }\end{array}$ & $-0.0003(0.993)$ & $-0.0540(0.001)$ & $-0.0487(0.012)$ & $-0.0390(0.092)$ & $-0.1130(0.000)$ & $-0.1025(0.000)$ \\
\hline Injection type (IV) & $-0.6554(0.000)$ & $-0.4325(0.000)$ & $-0.4659(0.000)$ & $-0.4888(0.000)$ & $-0.1243(0.005)$ & $-0.0315(0.477)$ \\
\hline $\begin{array}{l}\text { Injection-who } \\
\text { (HCP at clinic) }\end{array}$ & $0.4365(0.000)$ & $0.0141(0.772)$ & $-0.0436(0.442)$ & $0.3006(0.000)$ & $0.5339(0.000)$ & $0.2982(0.000)$ \\
\hline Observations & 1152 & 4000 & 2976 & 2176 & 4896 & 4640 \\
\hline Log likelihood & -331.0619 & -1240.7173 & -921.5068 & -654.4368 & -1481.2026 & -1470.9213 \\
\hline $\begin{array}{l}\text { Swait - Louviere } \\
\text { LL Ratio Test } \\
\text { Statistic }\end{array}$ & -27.91 & & -19.58 & & -20.74 & \\
\hline Results & $\begin{array}{l}\text { Exceeds test statisti } \\
\text { deemed different }\end{array}$ & c, so samples are & $\begin{array}{l}\text { Exceeds test statisti } \\
\text { deemed different }\end{array}$ & & $\begin{array}{l}\text { Exceeds test statisti } \\
\text { deemed different }\end{array}$ & c, so samples are \\
\hline
\end{tabular}

Bold values indicate statistical significance at the 0.05 level

$A E$ adverse events, $H C P$ healthcare provider, $I V$ intravenous, $P A S I 9090 \%$ reduction in the Psoriasis Area and Severity Index score 
IV injection type for both groups, as well as dislike of frequent injections. For patients, subgroup analysis does not find major differences between those whose psoriasis is mild and those whose psoriasis is moderate or severe. The only exceptions are the preference against IV injection which is more pronounced for those patients with a mild form of psoriasis. Also, patients with more severe disease are more tolerant regarding adverse events.

This finding compares with a recent study from the US in which risk of adverse events was more important than efficacy among moderate psoriasis patients, but results were the other way round for severe psoriasis patients [21]. In a German patient population, participants with moderate-to-severe psoriasis were most interested in safety of biologicals, followed by efficacy, but preferences varied with socio-demographic characteristics and working status [29]. Seston et al. [30] observed that psoriasis patients found the greatest benefit in low risks of skin cancer and liver damage, and preferred treatments that achieve moderate improvement more quickly. The treatment process attributes were of moderate importance in that study. Given the extreme risk aversion found in Japanese society [31], we also expected that Japanese patients would place a higher emphasis on the reduction of adverse events and have a smaller preference for efficacy.

Further, we found that, surprisingly, patients prefer to have their treatment administered in a clinic rather than at home. This is in a stark contrast to the findings of Schaarschmidt et al. [20], who found that psoriasis patients considered treatment location at home as the most important attribute for selecting psoriasis treatments. A possible explanation for this may be the risk aversion of the Japanese people coupled with the strong transportation networks making hospital access relatively easy. The risk aversion to potential mistakes in self-administered injections is traded off against the convenience of treatment at home, with different populations having different priorities. Another proposed explanation is the finding in other literature that a large proportion of Japanese people aged between 50 and 70 years are lonely [32], particularly when compared with Western countries [33]. Visiting a physician's office might be a strategy to cope with loneliness and a study has demonstrated a link between the desire to communicate with other patients in the waiting room and the number of office visits [34]. In line with this reasoning is the fact that Japan (and Korea) have by far the highest numbers of doctor's office consultations among OECD countries [35].

Considering the physician perspective, we find a strong aversion to the administration of treatment via IV injection. Otherwise, efficacy and avoidance of adverse events are the dominant attributes, which mirrors findings from a recent study in the US [21]. Subgroup analysis reveals that dermatologists place slightly less emphasis on efficacy compared with GPs but give more priority to safety (risk of adverse events). GPs have the strongest preference to administer the medication at a clinic among all physicians, while location does not factor much in the dermatologist sample's treatment decision, with a statistically insignificant slight preference for self-administration. GPs also have the strongest aversion against IV injection type.

In general, risk tolerance and preference for efficacy is similar between physicians and patients in our study, while a UK study found that patients had a greater tolerance for adverse events compared with their physicians [36].

Preferences between physicians and patients are quite similar in our results, although this study was not explicitly designed to assess the concordance of preferences across patient and physician pairs. Instead of testing the alignment of patient/physician pairs, in this study patients and physicians responded independently from each other and concordance was assessed in terms of the relative importance of specific treatment attributes. While paired comparisons are an interesting methodological approach, the majority of studies analysing the concordance of patient and physician preferences still rely on separate sample DCEs [37]. However, a recent Japanese study explicitly evaluated treatment goal alignment between psoriasis patients and their paired physicians. In that study, treatment goal misalignment was found in $67.9 \%$ of the patient/physician pairs. The misalignment was mainly 'patient predominant' $(60.9 \%)$, indicating that patients had higher expectations regarding 'complete clearance' than physicians expected [38]. In a German study that compared concordance between physicians and patients, patients worried more about mild adverse events and treatment location than the physicians, but relatively less about cost and frequency of laboratory tests [22].

Therefore, shared decision making (SDM) should be an integral part of psoriasis treatment [13], as SDM has been shown to positively impact patient satisfaction, treatment compliance and ultimately health outcomes [39]. Recent research showed that SDM is as important for Japanese patients as it is for Americans [40].

Limitations of this study include that this is a stated preference study such that the results rely on physician and patient participants being able to judge treatment options among the hypothetical options presented. Also, the only currently available treatment administered by IV injection is infliximab, so that attribute may be overly associated with this specific treatment.

Among the patient sample, the current use of biologics was low (13.1\%) and so we anticipate few had experience 
with this option and may not have been as familiar with the reality of this treatment option. Furthermore, the biologics being developed are generally intended for patients with more severe disease who are not being successfully treated with other approaches, including other systemics, so the patient sample may be somewhat broader than the intended patient pool.

\section{Conclusions}

This study provides insight into the treatment preferences of physicians and patients in Japan for treatment attributes for moderate and severe psoriasis. This includes both the direct expected health outcome attributes and process attributes in administering the treatment. Optimal communication between patient and physician regarding the decision-making process in treatment selection is crucial to patient-centred care and to ensure that patient preferences and physicians' perceptions are in concordance.

Data Availability Statement The datasets generated during and/or analysed during the current study are available from the corresponding author on reasonable request.

Author Contributions TB contributed to the concept of this study, design of the analysis, analysis of the data and the writing of the manuscript. HK contributed to the concept of the study and the writing of the manuscript. JM contributed to the concept of this study, design of the analysis, collection of data, the writing of the manuscript and supervision of this study.

\section{Compliance with Ethics Standards}

The study was approved by the sponsor's internal approval committee and was in accordance with ethical and legal guidelines for Japan. The data was collected through a web-based panel and individual identifying information was not obtained. Participating respondents were informed of the study purpose and informed consent was obtained from all individual respondents included in the study.

Funding This study's data collection was funded by Janssen Pharmaceutical.

Conflict of interest TB has no conflicts of interest. HK is an employee of Janssen Pharmaceutical. JM is an employee of Janssen Pharmaceutical.

Open Access This article is distributed under the terms of the Creative Commons Attribution-NonCommercial 4.0 International License (http://creativecommons.org/licenses/by-nc/4.0/), which permits any noncommercial use, distribution, and reproduction in any medium, provided you give appropriate credit to the original author(s) and the source, provide a link to the Creative Commons license, and indicate if changes were made.

\section{References}

1. Kubota K, Kamijima Y, Sato T, et al. Epidemiology of psoriasis and palmoplantar pustulosis: a nationwide study using the Japanese national claims database. BMJ Open. 2015;5:e006450.

2. Rachakonda T, Schupp C, Armstrong A. Psoriasis prevalence among adults in the United States. J Am Acad Dermatol. 2014;70(3):512-6.

3. Parisi R, Symmons D, Griffiths C, Ashcroft D. Global epidemiology of psoriasis: a systematic review of incidence and prevalence. J Investig Dermatol. 2013;133(2):377-85.

4. Armstrong AW, Harskamp CT, Armstrong EJ. The association between psoriasis and obesity: a systematic review and metaanalysis of observational studies. Nutr Diabetes. 2012;2(12):e54.

5. Barrea L, Savanelli MC, Di Somma C, et al. Vitamin D and its role in psoriasis: an overview of the dermatologist and nutritionist. Rev Endocr Metab Disord. 2017;18(2):195-205.

6. Bhosle MJ, Kulkarni A, Feldman SR, Balkrishnan R. Quality of life in patients with psoriasis. Health Qual Life Outcomes. 2006;4:35.

7. Kopp T, Riedl E, Bangert C, et al. Clinical improvement in psoriasis with specific targeting of interleukin-23. Nature. 2015;521:222-6.

8. Florek A, Wang C, Armstrong A. Treatment preferences and treatment satisfaction among psoriasis patients: a systematic review. Arch Dermatol Res. 2018;310(4):271-319.

9. Sruamsiri R, Iwasaki K, Tang W, Mahlich J. Persistence rates and medical costs of biological therapies for psoriasis treatment in Japan: a real-world data study using a claims database. BMC Dermatol. 2018;18:5.

10. Takeshita J, Gelfand J, Li P, Pinto L, Yu X, Rao P, Viswanathan $\mathrm{H}$, Doshi J. Psoriasis in the US Medicare Population: prevalence, treatment, and factors associated with biologic use. J Investig Dermatol. 2015;135(12):2955-63.

11. Imafuku S, Nakano A, Dakeshita H, Li J, Betts K, Guerin A. Number needed to treat and costs per responder among biologic treatments for moderate-to-severe plaque psoriasis in Japan. J Dermatol Treat. 2018;29(1):24-31.

12. Lebwohl M, Kavanaugh A, Armstrong AW, Van Voorhees AS. US perspectives in the management of psoriasis and psoriatic arthritis: patient and physician results from the population-based multinational assessment of psoriasis and psoriatic arthritis (MAPP) survey. Am J Clin Dermatol. 2016;17:87-97.

13. Ashcroft D, Seston E, Griffiths C. Trade-offs between the benefits and risks of drug treatment for psoriasis: a discrete choice experiment with UK dermatologists. Br J Dermatol. 2006;155(6):1236-41.

14. Jenkins V, Catt S, Banerjee S, Gourley C, Montes A, SolisTrapala I, Monson K, Fallowfield L. Patients' and oncologists' views on the treatment and care of advanced ovarian cancer in the UK: results from the ADVOCATE study. Br J Cancer. 2013;108(11):2264-71.

15. Mühlbacher A, Stoll M, Mahlich J, Nübling M. Evaluating the concordance of physician judgments and patient preferences on AIDS/HIV therapy - a discrete choice experiment. Health Econ Rev. 2013;3:30.

16. Mühlbacher A, Stoll M, Mahlich J, Nübling M. Patient preferences for HIV/AIDS therapy-a discrete choice experiment. Health Econ Rev. 2013;3:14.

17. Bolt T, Mahlich J, Nakamura Y, Nakayama M. Hematologists' preferences for first-line therapy characteristics for multiple myeloma in Japan: attribute rating and discrete choice experiment. Clin Ther. 2018;40:296-308.

18. Nakayama M, Kobayashi H, Okazaki M, Imanaka K, Yoshizawa K, Mahlich J. Patient preferences and urologist judgments 
on prostate cancer therapy in Japan. Am J Men's Health. 2018;12(4):1094-101.

19. Umar N, Schaarschmidt ML, Schmieder A, Peitsch WK, Schollgen I, Terris DD. Matching physicians' treatment recommendations to patients' treatment preferences is associated with improvement in treatment satisfaction. J Eur Acad Dermatol Venereol. 2013;27(6):763-70.

20. Schaarschmidt ML, Schmieder A, Umar N, et al. Patient preferences for psoriasis treatments: process characteristics can outweigh outcome attributes. Arch Dermatol. 2011;147(11):1285-94.

21. Alcusky M, Lee S, Lau G, et al. Dermatologist and patient preferences in choosing treatments for moderate to severe psoriasis. Dermatol Ther. 2017;7(4):463-83.

22. Schaarschmidt M-L, Herr R, Gutknecht M, Wroblewska K, Gerdes S, Sticherling M, et al. Patients' and physicians' preferences for systemic psoriasis treatments: a nationwide comparative discrete choice experiment (PsoCompare). Acta Dermato Venereol. 2018;98(2):200-5.

23. Coast J, Al Janabi H, Sutton EJ, Horrocks SA, Vosper AJ, Swancutt DR, Flynn TN. Using qualitative methods for attribute development for discrete choice experiments: issues and recommendations. Health Econ. 2012;21(6):730-41.

24. Lancsar E, Louviere J. Conducting discrete choice experiments to inform healthcare decision making. Pharmacoeconomics. 2008;26(8):661-77.

25. Kuhfeld WF. Marketing research methods in SAS experimental design, choice, conjoint and graphical techniques. Cary: SAS Institute; 2010

26. Johnson FR, Lancsar E, Marshall D, Kilambi V, Mühlbacher A, Regier DA, Bresnahan BW, Kanninen B, Bridges JF. Constructing experimental designs for discrete-choice experiments: report of the ISPOR conjoint analysis experimental design good research practices task force. Value Health. 2013;16(1):3-13.

27. Johnson R, Orme B. Getting the most from CBC. Sequim: Sawtooth Software Research Paper Series, Sawtooth Software. 2003.

28. Swait J, Louviere J. The role of the scale parameter in the estimation and comparison of multinomial logit models. J Market Res. 1993;30:305-14.

29. Kromer C, Peitsch W, Herr R, Schmieder A, Sonntag D, Schaarschmidt M. Treatment preferences for biologicals in psoriasis: experienced patients appreciate sustainability. J Dtsch Dermatol Ges. 2017;15(2):189-200.
30. Seston EM, Ashcroft DM, Griffiths CEM. Balancing the benefits and risks of drug treatment: a stated-preference, discrete choice experiment with patients with psoriasis. Arch Dermatol. 2007;143(9):1175-9.

31. Synodinos N. Understanding Japanese consumers: some important underlying factors. Jpn Psychol Res. 2001;43(4):235-48.

32. van den Broek T. Gender differences in the correlates of loneliness among Japanese persons aged 50-70. Australas J Aging. 2017;36(3):234-7.

33. Nicolaisen $\mathrm{M}$, Thorsen $\mathrm{K}$. Who are lonely? Loneliness in different age groups (18-81 years old), using two measures of loneliness. Int J Aging Hum Dev. 2014;78:229-57.

34. Hafner P, Mahlich J. Determinants of physician's office visits and potential effects of co-payments: evidence from Austria. Int J Health Plan Manag. 2016;31(3):e192-203.

35. OECD 2018. Doctors' consultations (indicator). https://doi. org/10.1787/173dcf26-en. (Accessed on 03 May 2018).

36. Gonzalez J, Johnson F, McAteer H, Posner J, Mughal F. Comparing preferences for outcomes of psoriasis treatments among patients and dermatologists in the UK: results from a discretechoice experiment. Br J Dermatol. 2017;176(3):777-85.

37. Harrison M, Milbers K, Hudson M, Bansback N. Do patients and health care providers have discordant preferences about which aspects of treatments matter most? Evidence from a systematic review of discrete choice experiments. BMJ Open. 2017;7:e14719.

38. Okubo Y, Tsuruta D, Tang A, Inoue S, Torisu-Itakura H, Hanada T, Ohtsuki M. Analysis of treatment goal alignment between Japanese psoriasis patients and their paired treating physicians. J Eur Acad Dermatol Venereol. 2018;32(4):606-14.

39. Renzi C, Di Pietro C, Gisondi P, et al. Insufficient knowledge among psoriasis patients can represent a barrier to participation in decision-making. Acta Dermato Venereol. 2006;86(6):528-34.

40. Schaede U, Mahlich J, Nakayama M, Kobayashi H, Takahashi Y, Saito K, Uemura H, Tokumitsu M, Yoshizawa K. Shared decisionmaking in prostate cancer treatment in Japan: patient preferences versus physician perceptions. J Glob Oncol. 2018;4:1-9. 